\title{
Water and life
}

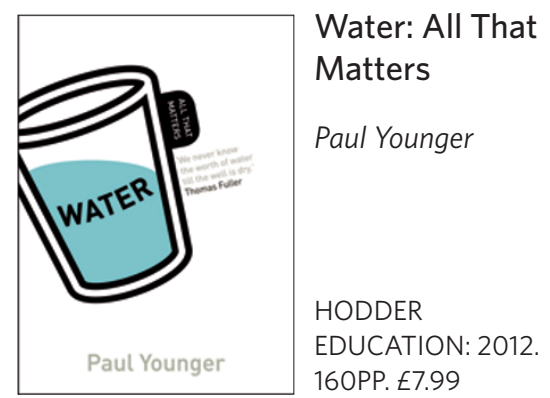

ater is one of our most precious
natural resources. A growing
human population, and the expansion of economic activities that comes with it, will intensify the pressure on water resources. As part of the All That Matters series, Paul Younger's book on water addresses this topic, and presents an allround introduction to the scientific, political and societal issues associated with this fundamental liquid.

Well written and exhaustive within the limits offered by the brief format of the series, Water: All That Matters attains the goal of summarizing all that is essential to know about water, and more: water management is presented as an integral part of the more general concern about natural resources. I would recommend the book to students in management and the hydrological or political sciences who are looking for a clear description of water management and the terrestrial water cycle.

Younger begins by reminding us of the remarkable chemical properties of $\mathrm{H}_{2} \mathrm{O}$, which together account for the presence of life on Earth. That water changes state - from frozen to liquid and from liquid to gaseous at 0 and $100{ }^{\circ} \mathrm{C}$, respectively ensures the predominance of its liquid form on Earth. And the dipolar nature of liquid water molecules, a result of the net charges of the oxygen and hydrogen atoms as well as the molecule's structure, leads to an electrical attraction between molecules that has three crucial effects: first, tight packing of water molecules in a liquid means less evaporation of water from the Earth's surface. Second, capillary action, where water rises through a narrow pipeline defying gravity, is essential for transport in the soil or in the stems of plants. And finally, the remarkable ability of water to dissolve other substances makes it the basic ingredient for life on Earth.

Two-thirds of Earth's surface is covered with oceans. Fresh water, however, only represents about $3 \%$ of the total amount of water on Earth - a planet with "few freshwater lakes and even fewer rivers to be readily spottable from space," as Younger puts it. More than two-thirds of this fresh water is frozen, stored in glaciers, ice caps and ice sheets. Of the volume of liquid fresh water, $99 \%$ exists as groundwater, so only $1 \%$ is directly accessible at the surface, in rivers, reservoirs and lakes.

The principles of hydraulics, and of the conservation of mass and energy, are presented to illuminate how water circulates. Even though perhaps conservation of momentum - that is, mass multiplied by velocity - may be better suited for this purpose than energy conservation, the chapter clearly outlines the concept of buoyancy: why some things float and others sink. Younger simply but comprehensively describes how water movement is governed in various types of water bodies. Rivers are principally controlled by friction and slopes. Lakes and wetlands are governed by the balance between inflows and outflows, and groundwater movement is determined by flow through a porous medium, viscosity and pressure gradients. Key aspects of the global ocean circulation are outlined concisely, too, including some of its complexities such as the role of the oceans in the Pacific climate seesaw El Niño, or the impact of sea surface temperatures on drought and floods.

Water means life. Paul Younger next delves into the wide variety of life forms hosted by water bodies. Aquatic life can, of course, be found in the open water. But life is just as rich in the bed sediments, where the majority of invertebrates dwell - and serve as food for fish and specialized birds. Younger reminds us that the "abundance, diversity and condition [of aquatic life] are excellent indicators of the overall health of a river". Life has also been identified in the terrestrial subsurface: invertebrates and microbes populate the groundwater; and estuaries, where surface and groundwater mix with sea water, are particularly diverse. But the open ocean remains the largest pool of biodiversity. As Paul Younger bemoans, aquatic life is under the constant threat of the actions of humanity, such as depletion of water resources, destruction of ecosystems, overfishing practices and anthropogenic climate change.

Water management is discussed at the end of the book. The use and reuse of water resources for human activities strongly varies: agriculture is the main use in developing countries, whereas much water consumption is dedicated to industry and energy production in developed regions. Climatic conditions are an important factor, too: water supply is a critical challenge for large cities in arid areas, and many regions of the world are affected by droughts of varying intensities, as a result of overpopulation, poor land-use practices, or local reductions of rainfall. As water is becoming scarce, the concept of a 'water footprint' has emerged, reflecting the total volume of fresh water that is used to produce goods and services consumed. Younger notes large disparities between countries, with an average of 7,790 litres per person per day for the USA, contrasting with only 1,500 litres per person per day in the Democratic Republic of Congo. These numbers are context-dependent and must be interpreted with care, but they illustrate the impact of humankind on water resources.

It is becoming increasingly difficult to manage our water resources, with the droughts and floods, the vulnerability of low-lying lands to sea-level rise, and the reductions in soil fertility that come with climate and land-use change. Political tensions over the right allocation of water result. Beyond the basic and commonly accepted principle that every human needs access to a sufficient amount of water for drinking and sanitation, Younger discusses how much personal water is an unrestricted right, who will pay for water and sanitation services, and whether the provision of water and sanitation should be national, private or semi-private.

Because of the connectivity between water and land use, water and carbon, and water and energy, solutions for integrated land and water resources management will emerge and become more and more effective. Paul Younger concludes that "the twenty-first century is to turn out more happily for humankind and ecosystems than the previous century did" - but only if water becomes a political priority. We must hope that it will.

\section{REVIEWED BY FRÉDÉRIC FRAPPART}

Frédéric Frappart is at Géosciences Environnement Toulouse, Observatoire Midi-Pyrénées, 14 Avenue Edouard Belin, F-31400 Toulouse, France. e-mail: frederic.frappart@get.obs-mip.fr

Published online: 2 December 2012 